\title{
Role of maternology in functional gastrointestinal disorders in infant
}

\author{
Daniela Marincas ${ }^{1,2}$, Simina Angelescu ${ }^{3}$, Coriolan Ulmeanu ${ }^{1,4}$ \\ 1"Carol Davila" University of Medicine and Pharmacy, Bucharest, Romania \\ ${ }^{2}$ Stella Maris SRL, Family Medicine, Bucharest, Romania \\ ${ }^{3}$ Angelescu Simina, privat psychology, Bucharest, Romania \\ ${ }^{4}$ Departament of Pediatrics, "Grigore Alexandrescu" Emergency Children's Hospital, Bucharest, \\ Romania
}

\begin{abstract}
The functional digestive pathology of the child in the first year of life is frequently encountered in the medical practice and is difficult to manage, diagnose and treat. The current general medical approach does not treat mother and child in the first year after birth as a biological unit, thus leaving out the perspective of the association of functional gastrointestinal disorders (FGID) with an impairment of maternal-child emotional relationship, resulting in uncertain therapeutic results. This perspective of the problem has been approached by maternology, a newer branch of medical sciences, which has been born on the assumption that most of the handicaps stem from a relational difficulty between the mother and her infant. Maternology integrates the child's suffering from the perspective of the emotional relationship of the parent-child couple, emphasizing that the mother and the child is a biological unit that needs to be diagnosed and treated together, thus giving a new view in addressing the baby's functional sufferings.
\end{abstract}

Keywords: functional gastrointestinal disorders in infant, maternology, mother-child emotional relationship

\section{INTRODUCTION}

The functional digestive pathology of the child in the first year of life is frequently encountered in medical practice. Functional gastrointestinal disorders (FGID) represent a group of disorders which are difficult to manage from a diagnosis and treatment standpoint.

FGID symptoms cause great discomfort for infants and parents, increasing the number of unnecessary physician presentations and healthcare costs. International expert committees meet periodically at the Rome Foundation to establish the latest developments and changes in the symptom-based classification system of FGID. FGID results from complex and reciprocal interactions between biological, psychological and social aspects (brain-gut axis) $(1,2,3,4)$.
It is mentioned in the literature that there is a link between parents' psychological state (anxiety, depression) and abdominal symptomatology in children, and also that the management of the most common FGID, such as infantile colic and regurgitation, should focus on education and parent reassurance, and nutritional advice, such as recommendations on volume, frequency, feeding techniques $(1,5)$.

However, some infants continue to suffer, which certainly shows that there may be other issues that escape the current medical approach.

An important percentage of this type of pathology is possibly influenced by the mother-child emotional relationship in the peak vulnerability period, which is the first year of life. There are manifestations described in psychology as psychoso- 
matic, and in maternology they are called "disorders of birth" $(13,15)$.

\section{The current medical approach to the problem}

The current general medical approach does not treat mother and child as a biological unit in the first year after birth, thus neglecting the perspective of the FGID link with the emotional mother-child relationship. Although there are many papers about the emotional plan, the parent, which in this case is the mother, is considered to be only a caregiver of the sick child, who needs encouragement and support for the concern that arises because of the baby's health issues, but it is not clear if there is a relationship between the mother's condition and the baby's symptoms (6). The functional impairment of the infant often persists despite any conventional treatment, without explanation, beyond the time limits suggested in the scientific literature (7).

Over the time, FGID have generated various diagnostic and treatment problems for practitioners, as the diagnosis required the exclusion of organic and inflammatory pathologies. If initially FGID were considered to be less scientifically explicable, less treatable, largely considered psychiatric, only in the last three decades of the 20th century deep studies were carried out, these studies discovered links between mind and body, making them parts within a common system. Thus, the concept of the biopsychosocial model of diseases (1977, George Engel) was born. In the biopsychosocial model, gastrointestinal functional symptoms integrate: motility disorders, increased visceral sensitivity, under conditions of coordination between the brain and the gastrointestinal tract in the presence of influences from psychosocial factors.

FGID are an important group of diseases, currently accepted as self-contained medical entities, both in practice and in medical research.

Since 1992, international expert committees have been set up to clarify the FGID and have begun developing a common international classification of FGID using the symptom-based classification system. The Rome Foundation is the current international organization that sets out the criteria for diagnosing and classifying FGID based on symptoms (7). The Rome IV consensus on diagnostics and classification criteria for digestive functional disorders for the pediatric side has maintained the two major categories: neonatal and infant disorders and disorders of older children and adolescents.
The GI disorders in infants and young children according to the Rome IV classification are:

G.1. Infant regurgitation

G.2. Infant rumination syndrome

G.3. Cyclic vomiting syndrome

G.4. Infant colic

G.5. Functional diarrhea

G.6. Infant dyschezia

G.7. Functional constipation (7)

\section{The current state of knowledge}

It is known that during childhood, the structure and function of the gastrointestinal tract (GI), nervous system and microbiota is still maturing and this can cause signs and symptoms of GI that do not have a clear structural or biochemical cause. Diagnosis of a functional disorder virtually eliminates organic disease as a cause of symptoms. Regurgitation, infantile colic and functional constipation are the most common FGID in childhood, and it has been shown that more FGID can coexist often in the same child. Infant dyschezia, functional diarrhea, infant rumination syndrome and cyclic vomiting syndrome occur less frequently $(4,5)$.

\section{G.1. Infant regurgitation}

Infant regurgitation is the most common FGID in the first year of life and may occur in healthy infants between three weeks and one year of age. Recognizing infant regurgitation can avoid frequent visits to physicians and unnecessary investigations, as well as therapy for gastroesophageal reflux, a disease that can often be confused with infant regurgitation. But we are also usually dealing with an anxious mother, who frequently turns to a pediatrician, because she worries not only about the child's condition but also about her own condition, a state she certainly does not wish to talk about (5). It has been noticed that interaction between physician and the child's mother/caregiver often helps to improve the latter's condition. It is said that parents are concerned about the condition of their child, the identification of the sources of physical and emotional suffering, and the plans to eliminate stressful conditions (5).

\section{G.2. Rumination}

Rumination is the regular regurgitation of stomach contents in the mouth for self-stimulation. Infant rumination syndrome is a disorder which is rarely described in literature. Maternal behavior associated with this syndrome can either be negligent or extremely attentive, but there seems to be a lack of pleasure in holding the baby or the lack sensitivity to the child's needs for comfort and satisfaction 
$(5,7,18)$. The treatment aims to help parents address their feelings about the child, and improve their ability to recognize and respond to children's physical and emotional needs (5).

\section{G.3. Cyclic vomiting syndrome}

Data on clinical evolution in infants and young children are rare, epidemiological studies report that they may occur before the age of three (5).

\section{G.4. Infant colic}

Understanding colic in infants requires an appreciation of the child's development, the diadic relationship of the caregiver with the child, and the family and social environment in which they exist (7). Infant colic has been described as a behavioral syndrome in infants aged one to four months which involves long crying periods and an unrested behavior which is hard to calm down. There is talk in the literature about the need for a better assessment of parents' vulnerabilities, such as depression, lack of social support, and the necessity for the parents' continued availability for the family. If the attempts to control a child's crying fail, it might come to anxiety, frustration, exhaustion, mother's doubts about her competence to care, contradictory feelings, or even alienation to the uncomfortable child which can increase the risk for the appearance of "shaken child syndrome" and other possible forms of abuse (5).

\section{G.5. Functional diarrhea}

Functional diarrhea is defined by the recurrent daily painless passage of three or more unformed large stools for four or more weeks with onset in the early childhood or preschool. There is no need for medical intervention, but effective reassurance of parents is important (5).

\section{G.6. Infant dyschezia}

Infant dyschezia is defined as a visible effort to defecate, screaming and crying for many minutes. In most infants, the symptoms begin in the first few months and resolve towards the end of the first year of life. In terms of treatment, the parents of the child often need reassurance about the absence of a pathological process requiring medical intervention (7).

\section{G.7. Functional constipation}

Functional constipation has a low prevalence in the first year of life. Physical examination is important for both the clinician and the parents to ensure that there is no illness. Symptoms usually improve with the onset of the intervention (daily volume laxatives to soften the stool) (5).

FGID symptoms may take forms from mild to extremely severe for the child and parents, and can induce parental anxiety, poor quality of life, with short- and long-term health consequences, shortening the duration of exclusive breastfeeding, frequent medical consultations and care, raising the costs unreasonably.

A more recent review of the literature has shown that there is a clear impact of FGID symptoms on family life and on short-term and long-term wellbeing, studies have reported that inconsolable crying and baby colic in the first three months of life are associated with lack of sleep and fatigue in mothers or even postpartum depressive symptoms. There has also been observed an inadequate mother-child interaction, a mother-insecure child attachment, concomitantly with feeding problems and frequent changes in formulas, all being associated with disconsolate crying fits (5).

What appears in the literature as a vicious circle, in which the continued suffering of the child leads to contradictory or estranged feelings towards the child by the caregiver, may also be an initial maternal disorder that results in a functional digestive problem to infant.

More recent research into the interaction between mothers and infants has demonstrated that there is a close link between maternal mental health problems and the cognitive and behavioral performance of children (8).

Limited research has highlighted a link between children's health problems and the mother's insecure attachment style. It has lately been found that a significant number of new mothers suffer from depressive or anxiety symptoms; postpartum depression affecting $10-15 \%$ of new mothers $(9,11)$. Risk factors for postpartum depression have been studied in detail, including stressful life events, poor social support, depressive symptoms or anxiety during pregnancy, a history of depression, unpleasant childhood experiences, a family history of mood disorders. However, their consequences for childcare are still incompletely observed and understood. Unrecognized and untreated depressive symptoms have been found to result in significant psychological disability for mothers, and children are at risk of serious developmental, behavioral, and emotional problems $(9,10)$.

There are authors who argue that it is of major importance to address the influence of the maternal anxiety disorder in longitudinal studies in order to improve the knowledge of the possible consequences of development and interaction. There are talks about specific intervention strategies that could enhance maternal regulatory dyadic competence to prevent functional disorders during infancy and childhood (8). 
This perspective of the problem has been approached by maternology, which was born based on the hypothesis that most of the disabilities come from a relational difficulty between mother and her infant (except for genetic problems or accidents). Maternology was created to understand and treat psychological motherhood processes and its main objectives are the study of psychological maternity, the recognition and understanding of the maternal difficulties, the creation of the mother-child bond, the early prevention of developmental disorders and the prevention of maltreatment.

Maternology is a therapeutic approach of the psychological dimension of motherhood, focusing on the difficulties of the mother-child relationship after birth (11). It is a new medical discipline that originated in France since 1987 with the first maternology service (within the Hospital J.-M. Charcot of Saint-Cyr-L'Ecole, Yvelines) led by Dr. Jean Marie Delassus and his team, the term maternology being accepted as such in the French language in " Le Grand Robert de la langue française» in November 2001 .

The concepts of maternology allow for a better understanding of the ways of access to the mother, but especially the difficulties or obstacles that may prevent this access. At first glance, these concepts may appear obscure, unnecessary for the professionals who surround the woman, the future mother or the mother (11). Two of the concepts of maternology are essential to understanding the psychology of maternity: the concept of totality and the concept of dedication. They explain how and what organizes maternity, birth and the possibility of their existence. Due to its early sensory and motor capacities, the fetus records, from the very early stages of pregnancy, the homogeneity of the intrauterine life and experiences the continuous experience of totality, which can be defined as the ensemble of harmonious processes that dominate prenatal life and structures the child, which redefines its genetic heritage and creates a specific need to continue to live the life he lived before coming into the world. At birth there is a rupture between the conditions of homogeneity felt by the fetus as a prenatal totality and the total lack of this postnatally. The mother is the one that which is expected to make the junction between the prenatal totality and the world, which is to ensure the child's mental/psychological birth $(11,12)$.

The totality will be found in the relationship between mother and child, passing through as experience, from the mother who lives it to the child who needs it.
There is a natal cycle of giving, which replaces the one of fetal-placental exchanges, and which constitutes a natal world, because the child sends back to the mother what she has initially given. This cycle of giving begins very shortly after birth and structures the mother-child bond.

The act of giving is best and most profoundly done during the feeding of the child, which makes breastfeeding an essential psychological situation. The mother extracts the gift from her, or tries to do it, and this is already an intense moment. Then the gift goes to the child who can receive it, which most often returns it to the mother, creating an endless cycle. Human maternity is characterized by the presence of total or lacunar ability to give. In this, and not in a supposed inborn maternal instinct, there is a way of approaching and understanding maternity and psychological birth, both in their normal and pathological state (11).

The woman who brings a child into the world does not automatically become a mother in the psychological sense of the term. There may be disturbances in the relationship that hampers the psychological birth of the newborn and the mother's access to maternity. The mother's sufferings are often silent, women often disguising themselves for shame, for fear of being judged, or for desperation that they are not good mothers (16).

The question arises as to whether there are procedures that allow detection, following and diagnosing mother-child difficulties which could harm the baby's psychological birth. The mental dimension of maternity and its genesis requires a different approach and a specific medical field.

Maternology, the new branch of medical sciences created in France, brings to the forefront a reconsideration of the life of the fetus during pregnancy and the condition in which the fetus is at birth, the mother's history, starting from her own birth to the pre-birth of her child, an essential moment of birth-giving and, last but not least, the diagnosis and treatment of the difficulty of being a mother (in the first 9 months postnatally, ideally as early as possible).

Human maternity, which is essentially a psychological motherhood, goes through four stages of psychological development: constitution of the original, breaking of the syncretism, self-attribution of the mother, confirmation by the father. Mother's blocking at one of the above stages leads to the occurrence of maternal (maternal diseases) and child suffering (birth diseases) $(12,14)$. Maternal difficulty is not necessarily visible or expressed and may exist without signs; the state of the child 
and the child's abilities to establish a relationship might inform us more precisely. The child is "the little clinician" of maternology. Observing and accompanying the breastfeeding situation (breast / bottle feeding) is the key in establishing a diagnosis and initiating maternological therapy $(12,14)$.

The semiological repertoire includes numerous infant signs and symptoms, including digestive functional disorders such as infant colic, regurgitation, gastro-esophageal reflux, food denial, constipation, diarrhea, etc. TGIF in infants are frequent reasons for pediatric consultation / admission.

Clinical observation of the newborn and infant shows that the lack of transfer of totality from mother to child creates the possibility for birth diseases ("disorders of the psychological birth" or disnatality), on the child's side and maternal diseases, meaning the sufferings and difficulties of the mother(12,13). To be born psychically, the child needs to find the totality experienced in prenatal life, and experience it in the extrauterine life. If the child does not receive this experience and only his physical well-being is provided, then he cannot adapt to the environment, and his physical development and health will be threatened. We are dealing with what in maternology are called birth diseases, dysfunctions that can be an integral part and a new chapter in pediatrics $(11,13)$.

The child suffers as soon as he's born, but in compensation, there is a sensitive period of the cerebral cortex, with a certain plasticity of the brain, in which the bad beginnings can be corrected. Therefore, as far as the first year of life is concerned, it is imperative to have relevant observational means and relevant semiological elements (17).

The child arriving in the world suffers a collapse of the previous world and must be able to evolve, through investment by the representative of the original totality - the mother. If this investment is not provided, psychological birth is in jeopardy and therefore the child's existence itself. Superficially, it may look like depression, but it is actually the lasting onset of what is called "natal collapse" that takes place in various forms, and in maternology the term of birth diseases (or disnatality) is preferred $(12,14)$.

The critical element to remember is the giving cycle, which is obviously noticeable during nursing, whether it's breastfeeding or bottle feeding. When the mother gives or attempts to give, she experiences moods she cannot control because they can only be a reflection of her profound experience, while the child reacts, and thus the psychological birth of the child manifests $(12,13)$.
Through repeated videoclinical observation of the moment of nursing, there have been described three normal phases of breast / bottle feeding: absorption in which the child is focused, while his hunger is satiated; dialogue or relationship phase in which the child stops and looks for the mother's gaze, there is an exchange of glances and smiles between mother and child, and a two-way transfer takes place; reverie or dream phase, the baby remains active in the mother's arms and finishes the process of feeding. This brings about the psychological birth of both the child and the mother, in terms of the mother - child relationship, as well as the participation to the relationship with the surrounding world.

Filming, examining and analyzing the moment of breast / bottle feeding has repeatedly made it possible to observe the behavioral problems of mother - child duo at a very early stage, as well as to track their therapeutic evolution.

The originality of the maternological approach is to understand that behind what is obvious and visible there is something else, namely that the initial problem is that of the mother's. She is the most capable to create the intention that generates the psychological flow of totality, she must have the desire to give, to have the ability to give and to have the means to transfer the totality to the child; transfer is at the heart of this issue. Mother and child are linked through psychological birth, which needs for the mother to have the means to get through with it. Thus, birth and maternity diseases are treated together, but at the level of transfer between mother and child. Hence the need for a change in the medical approach $(15,17)$.

Maternological diagnosis is a complex medical approach which sits at the base of maternological therapy. In observing the parent-child relationship, the one to be observed is the child, also called the "little clinician," providing the least error-prone data. The therapy is specific, non-aggressive and performed in care groups. Video analysis of clinical the behaviors of the mother-baby duos and the diagnosis of the structuring level of the stages of maternogenesis leads the team of specialists together with the mother in her deep suffering and together with child in his natal difficulty. Mother's care in an adequate space and in psychotherapy sessions raises the mother's awareness, which can be saving both for her and her child. Early maternological approach reduces the risk of developing somatic pathologies in children $(11,12)$.

Birth is not always a simple event, but can be accompanied by suffering and difficulties affecting 
both the child and the mother. They have a common problem: the totality which the first needs to receive and which the latter needs to give. The likelihood of the delivery cycle must be ensured before the possibility is hijacked by disease. This is the subject of maternology, which is the medicine of the first bond.

\section{CONCLUSIONS}

The medical approach centered on the child and the treatment of his symptoms turns his mother into the caregiver of the sick child, which can be called pediatric avoidance syndrome.

The child's difficulty is replaced by that of the mother's, and the mother's ability to care takes the place of maternal competence, thus creating a pathological maternal-child relationship and a real obstacle to healing the child's health problem.

\section{REFERENCES}

1. Drossman DA Functional Gastrointestinal Disorders: History, Pathophysiology, Clinical Features, and Rome IV. Gastroenterology 2016;150:1262-1279

2. Van Oudenhove L, Levy RL, Crowell MD et al. Biopsychosocial Aspects of Functional Gastrointestinal Disorders: How Central and Environmental Processes Contribute to the Development and Expression of Functional Gastrointestinal Disorders. Gastroenterology 2016;150:1355-1367

3. Fodor I, Dumitrașcu DL. Patologia funcțională digestivă pediatrică la sugar și copilul mic. Ce aduce nou Roma IV. Viata Medicala 17 Martie 2017; Numărul 11 (1417)

4. Allen AP, Dinan TG, Clarke $G$ et al. A psychology of the human brain-gut-microbiom axis. Wiley Online Library Apr. 2017

5. Salvatore S, Abkari A, Cai W et al. Review shows that parental reassurance and nutritional advice help to optimise the management of functional gastrointestinal disorders in infant. Wiley Acta Paediatrica 2018 Sep; 107(9): 1512-1520

6. Vandenplas Y. Algorithms for Common Gastrointestinal Disorders. JPGN Volume 63, Supplement 1, July 2016

7. Benninga MA et al. Childhood Functional Gastrointestinal Disorders: Neonate/Toddler. Gastroenterology 2016;150:1443-1455
Maternal difficulty may suspend or prevent the psychological birth of the child, but the doctor and / or medical team can unlock the process - by supporting and encouraging mother's maternity.

Maternology integrates the child's suffering from the perspective of the emotional relationship of the parent-child couple emphasizing that the mother and the child is a biological unit that needs to be diagnosed and treated together, thus giving a new perspective in addressing the baby's functional sufferings.

Perinatal care specialists play a key role in preventing mother-child relationship disorders and therefore need to contribute in supporting and understanding psychological motherhood by accessing maternology information.

Conflict of interest: none declared Financial support: none declared

8. Reck C, Tietz A, Müller M et al. The impact of maternal anxiety disorder on mother-infant interaction in the postpartum period. PLoS One 2018

9. Akman I, Kuşçu K, Özdemir N et al. Mothers' postpartum psychological adjustment and infantile colic. BMJ Jornals 2006

10. Ulmer-Yaniv A, Djalovski A, Priel A et al. Maternal depression alters stress and immune biomarkers in mother and child. Depression and Anxiety-The official journal of ADAA, 2018

11. Delassus JM. Le Sens de la maternité, $3^{e}$ edition. Paris: Ed. Dunod, 1997, trad. Sensul maternității, laşi: Minied , 2015

12. Delassus JM., Carlier L, Boureau-Louvet V. L'aide mémoire de maternologie. Paris: Ed. Dunod, 2010

13. Delassus JM. Psychanalyse de la naissance. Paris: Ed. Dunod, 2008

14. Delassus JM. Le génie du fœtus. Paris: Ed. Dunod, 2001

15. Delassus JM. Penser la naissance. Paris: Ed. Dunod, 2011

16. Delassus JM. La difficulté d'être mère. Paris: Ed. Dunod, 2014

17. Delassus JM. Neuroscience de l'être humain. Paris: Encre Marine, 2012

18. Lebovici S. L'arbre de vie - Éléments de la psychopathologie du bébé. Éd. ERES, 2009 